\title{
Correlation of Ankle Brachial Index with Peripheral Vascular Disease in Type 2 Diabetes Mellitus
}

\author{
Saeed Razaq ${ }^{1}$, Khudija $\mathrm{Nayab}^{2}$, Mahrukh Fatima ${ }^{3}$ \\ 1,2. Post Graduate Trainee and Medical Officer Hayatabad Medical Complex Peshawar, Pakistan \\ 3. House Officer Bahawal Victoria Hospital, Bahawalpur \\ Corresponding Author: saeedrazaq@live.com
}

\begin{abstract}
Objective: The aim of this study was to correlate ankle brachial index (ABI) with peripheral vascular disease (PVD) in type 2 diabetes mellitus. Material and methods: This was a hospital based interdisciplinary prospective study. A total of 100 patients in the age group of 30 to 80 years having peripheral vascular disease with predefined inclusion criteria were included in the study. Patients with other causes of raised blood sugar or peripheral vascular disease were excluded by relevant investigations. Ankle Brachial Index (ABI) was calculated using sphygmomanometer, and Colour Doppler Ultrasound (CDU) was used to diagnose PVD. Both the ABI method and the CDU method were compared for detecting PVD. Results: In our study, mean age was $60.04 \pm$ 5.03 years, mean body mass index was $27.10 \pm 2.67 \mathrm{~kg} / \mathrm{m} 2$ and mean duration of diabetes was $7.75 \pm 1.50$ years. Among the 68 diagnosed cases of PVD on CDU, 70.6\% cases were detected to have PVD by the ABI method (true positive) whereas $20(29.4 \%)$ cases remained undiagnosed when ABI alone was used for the diagnosis (false negative). Conversely, among 51 diagnosed cases of PVD by ABI method $5.9 \%$ cases were found to be normal on CDU (false positive). The ABI method was found to have specificity of $88.5 \%$ but the sensitivity was only $70.6 \%$. Conclusion: ABI has a very high specificity but the sensitivity is low compared to colour Doppler ultrasound. Therefore, if ABI is abnormal, the diagnosis of PVD is almost certain but in symptomatic cases with normal ABI; colour Doppler should be performed to exclude the PVD.
\end{abstract}

Keywords: Ankle Brachial Index, peripheral vascular disease, Colour Doppler Ultrasound, type 2 diabetes mellitus.

DOI: $10.7176 / \mathrm{JMPB} / 57-02$

Publication date: July $31^{\text {st }} 2019$

\section{Introduction}

Diabetes, and its complications, have become an epidemic that has become an important and challenging health problem. By 2025, Pakistan will have the highest number of diabetes patients (57 million) out of the world total (300 million) 1 . One of the complications of uncontrolled diabetes mellitus is peripheral vascular disease (PVD)2 . It is also one of the main causes of amputation of lower limb3 that occurs 12 times more often in diabetics than those without it4. PVD is also a risk factor of foot infection5 . Both PVD and infections are among the major contributors of leg amputations, if a diabetic foot ulcer is present. Diagnosis of PVD among diabetic patients has its limitation due to diminished clinical manifestations of the disease and diagnostic methods. A high level of specificity $(83.3-99.0 \%)$ and accuracy $(72.1-89.2 \%)$ of the method in detecting $\geq$ $50 \%$ stenosis has been reported for an anklebrachial index $(\mathrm{ABI}) \leq 0.906$. ABI is a reproducible and reasonably accurate, non invasive measure for the detection of PVD and the determination of disease severity 7 . Therefore, in 2003, American Diabetes Association (ADA) recommended measuring ABI in all diabetic patients older than 50 years of age, and in younger patients with any other PVD risk factors (e.g., smoking, hypertension, hyperlipidaemia, or duration of diabetes $>10$ years) 8 . Most epidemiological studies have used ABI to diagnose PAD9 . ABI is cheap, reliable and non-invasive method for detecting PVD in type 2 diabetes mellitus. It can be used as an initial screening test for earlier detection of PVD. Materials and methods This was a hospital based interdisciplinary prospective study, approved by the institutional review board and informed consent was obtained from each individual. The study followed the tenets of the declaration of Helsinki. A total of 100 randomly selected cases of PVD with type 2 diabetes mellitus (as per American Diabetes 2011 criteria based on consensus opinion from National Data Diabetic Group and WHO) having predefined inclusion and exclusion criteria were studied over a period of one-andhalf-years. Patients of age $>14$ years with fasting blood sugar $\geq$ $126 \mathrm{mg} / \mathrm{dl}(7 \mathrm{mmol} / \mathrm{l})$ and post-prandial blood sugar after a $75 \mathrm{~g}$ oral glucose $\geq 200 \mathrm{mg} / \mathrm{dl}(11.1 \mathrm{mmol} / \mathrm{l})$ were included as cases whereas patients with age below $<14$ years, having metabolic syndrome and having other causes of raised blood sugar or PVD as excluded by relevant investigations were not included in the study. All the cases underwent detailed history and thorough general physical examinations with special emphasis on 
systemic quantification of PVD symptoms. Anthropometric measurement in the form of height, weight, BMI (Body Mass Index) and ABI were taken and recorded. Biochemical examination with standard laboratory technique in the form of $\mathrm{CBC}$, fasting and postprandial blood glucose estimation, urine examination, glycosylated haemoglobin estimation, fasting lipid profile, blood urea and serum creatinine estimation, liver function tests were also done to diagnose and quantify the diabetes and its severity. All the cases with PVD also underwent additional evaluations including Colour Doppler Ultrasound (CDU), ophthalmic examination, ECG, CT scan for cerebrovascular profile and coronary angiography for cardiovascular profile, sphygmomanometric measurement of ankle and brachial artery pressure, Doppler examination to auscultate and record blood flow from dorsalis pedis and posterior tibial and brachial arteries and Treadmill testing to assess functional limitations objectively (decline of ABI immediately after exercise provides further support of diagnosis of PVD). Ankle brachial index (ABI) was calculated for each leg. The ABI value was determined by taking the higher pressure of the 2 arteries at the ankle divided by the brachial arterial systolic pressure. In calculating the ABI, the higher of the two brachial systolic pressure measurements was used. In normal individuals, there should be a minimal (less than $10 \mathrm{mmHg}$ ) interarm systolic pressure gradient during a routine examination. A consistent difference in pressure greater than $10 \mathrm{mmHg}$ between the arms was suggestive of, and a difference greater than $20 \mathrm{mmHg}$ was diagnostic of subclavian or axillary arterial stenosis, which may be observed in individuals at risk for atherosclerosis. ABI $<0.9$ was the cut-off used for diagnosis of PVD. The statistical analysis software SPSS version 17 (IBM SPSS Statistics for windows, SPSS Inc., USA) was used to compare and analyse the data for all the cases.

\section{Results}

Among 100 cases included in the study, there were 61 males and 39 female patients with a male to female ratio of 3:2. The youngest patient with type 2 diabetes mellitus was 39-years-old whereas eldest was 80 -years-old, with a mean age of 60.04 years (Standard Deviation, SD 5.03). Majority of the cases were older than 50 years $(78 \%)$ and only $22 \%$ cases were below 50 years (Fig. 1). The mean duration of type 2 diabetes mellitus was 7.75 years (SD 1.50). Among all the cases, $22 \%$ cases had diabetes of less than 5 years of duration whereas only $2 \%$ cases had diabetes of more than 15 years duration (Fig. 2). The mean BMI was $27.10 \mathrm{~kg} / \mathrm{m} 2$ with standard deviation of 2.67. Among the cases, only $24 \%$ cases had BMI in the normal range; $36 \%$ of male and $29 \%$ of females were overweight, $9 \%$ were obese and $3 \%$ were very obese (Fig. 3 ). Among the cases, $51 \%$ were found to have peripheral vascular disease (PVD) detected by ABI method. Majority of the cases with PVD were above 50 years with only 1 case who had PVD in younger age group, i.e., less than 40 years of age. PVD was more common in those who had longer duration of diabetes mellitus, i.e., more than 5 years with majority having 5 to 15 years of diabetes mellitus (Figs. 4, 5 and 6). Among the cases, 6 subjects were found to have calcification of peripheral vessels detected by colour Doppler ultrasound (CDU) and they were not included while calculating for sensitivity and specificity of ABI. Out of the remaining 94 cases, 68 were diagnosed to have PVD on CDU and 51 cases were detected by ABI method. Among the 68 diagnosed cases of PVD on CDU, only 48 (70.6\%) cases were detected by the ABI method (true positive) whereas $20(29.4 \%)$ cases remained undiagnosed when ABI alone was used (false negative). Conversely, among 51 diagnosed cases of PVD by ABI method 3 (5.9\%) cases were found to be normal on CDU (false positive). Therefore, ABI was found to have very high specificity, but low sensitivity for detection of PVD (Table I) 


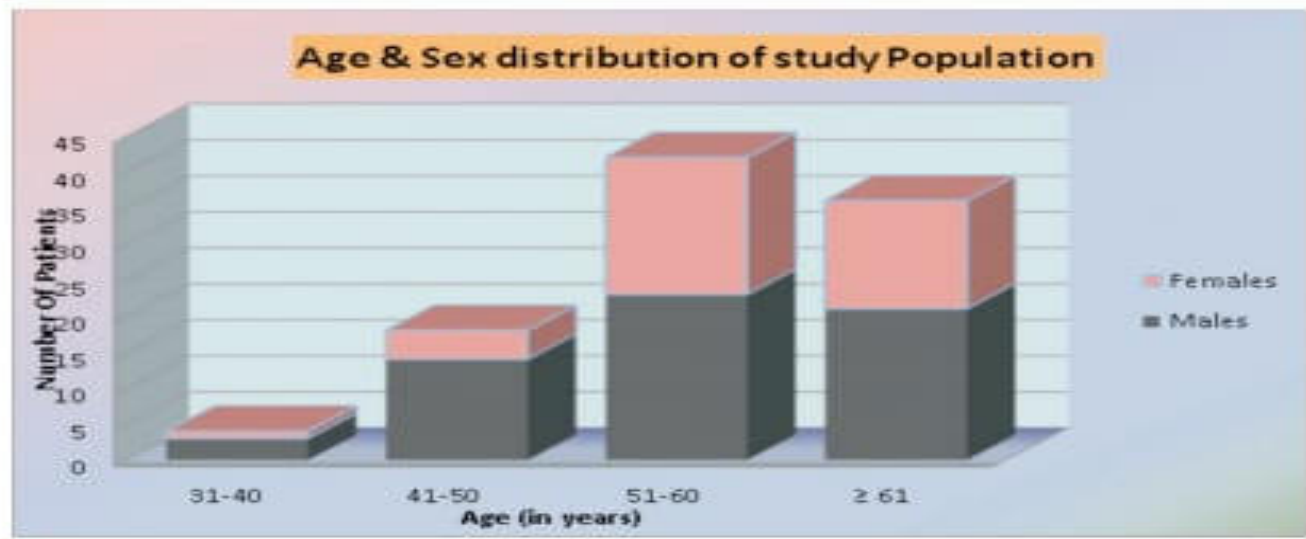

Fig. 1: Age and sex distribution of cases.

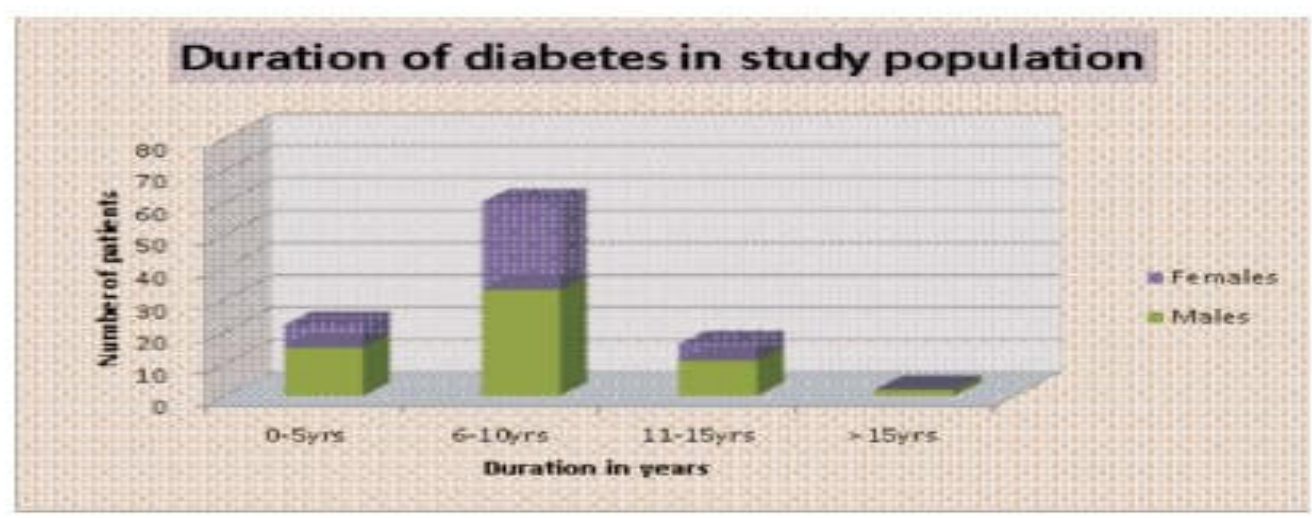

Fig. 2: Duration of diabetes mellitus in cases. 


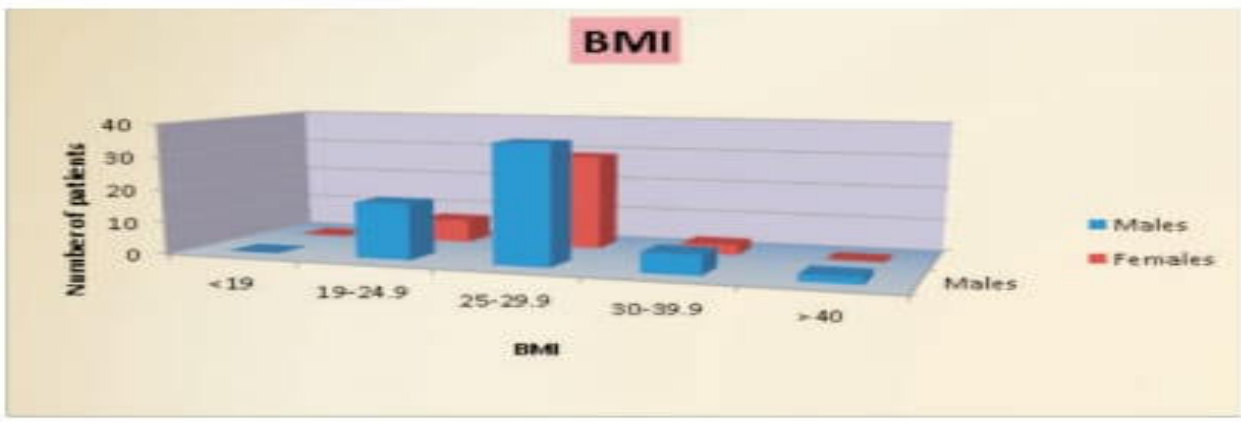

Fig. 3: Grading of BMI in cases.

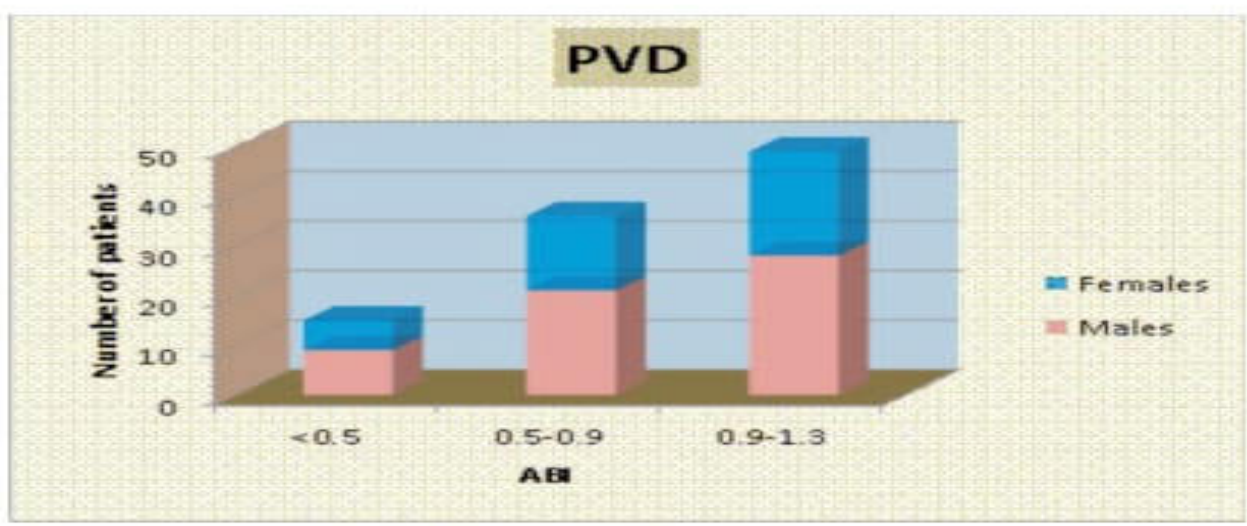

Fig. 4: Occurrence of PVD in cases using ABI.

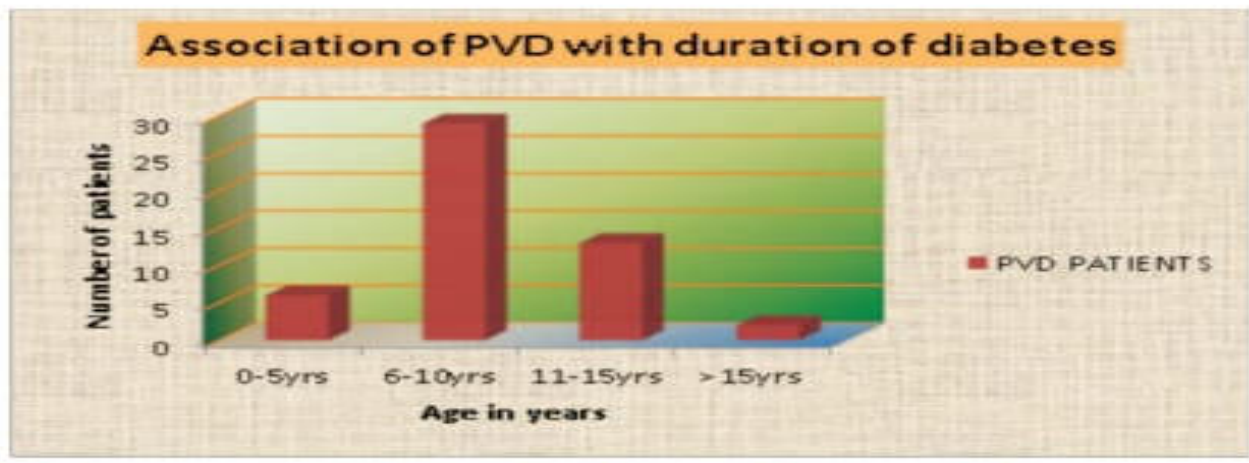

Fig. 5: Association of PVD with duration of diabetes mellitus. 


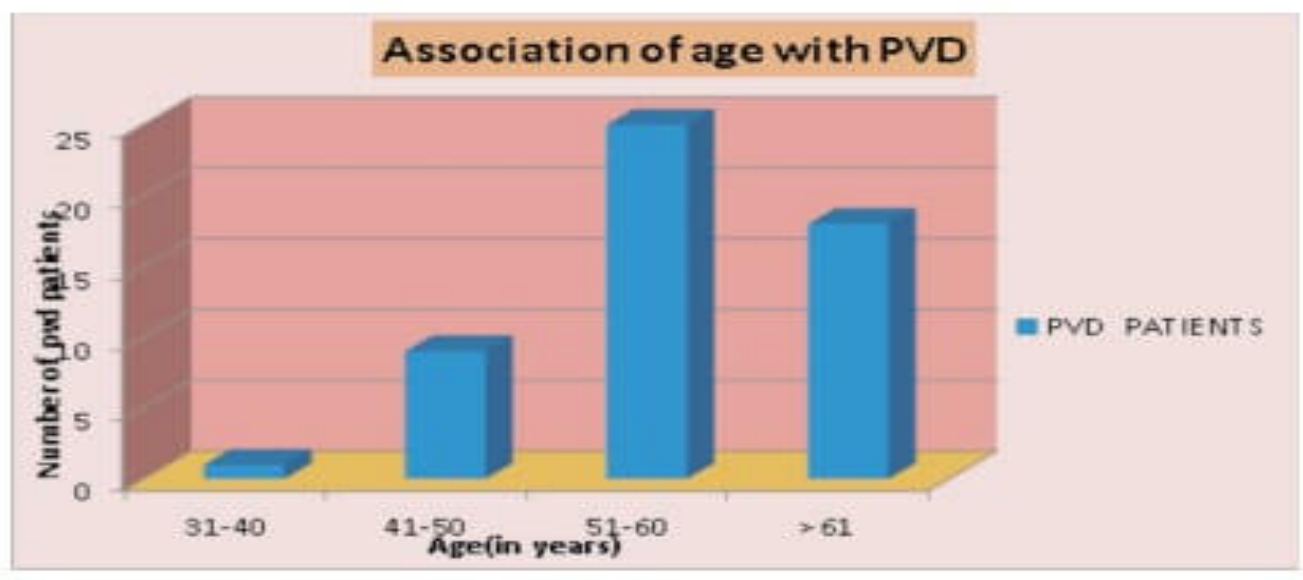

Fig. 6: Association of age with PVD.

Table I: Overall sensitivity and specificity of ABI vs colour Doppler ultrasound in PVD cases.

\begin{tabular}{lcc}
\hline CDU & \multicolumn{2}{c}{ ABI } \\
\hline (colour Doppler ultrasound) & Normal & Abnormal \\
\hline Normal & $23(\mathrm{TN})$ & 3 (FP) \\
\hline Abnormal & $20(\mathrm{FN})$ & $48(\mathrm{TP})$ \\
\hline
\end{tabular}

TP-True positive, FP-False positive, TN-Truenegative, FN-False negative.

Study of agreement between $A B I$ values and CDU findings showed a $100 \%$ agreement with CDU for the $A B I$ value of less than 0.5 whereas the $A B I$ value of 0.9 or more showed only $47.9 \%$ agreement with CDU (Table II).

Table II: Agreement between ABI and colour doppler ultrasound for PVD cases

\begin{tabular}{lccc}
\hline ABI & CDU & Total \\
\hline$>0.9$ & Normal & Abnormal & \\
\hline $0.7-0.9$ & 25 & 23 & 48 \\
\hline $0.5-0.7$ & 2 & 5 & 7 \\
\hline$<0.5$ & 0 & 30 & 30 \\
\hline Total & 0 & 15 & 15 \\
\hline
\end{tabular}

\section{Discussion}

The mean age of the cases in our study was $60.04 \pm 5.03$ years and the age range of the cases was from 30 to 80 years. This was comparable with the study of Premalatha et al10. The present study had more male diabetics (61) compared to females (39) with male to female ratio of 3:2. The mean duration of diabetes was $7.75 \pm 1.50$ years which is comparable to the study of Banait et al11 . In our study, the mean BMI was $27.10 \pm 2.67 \mathrm{~kg} / \mathrm{m} 2$ 
comparable to the study of Orchard et al12. As it is difficult to perform CDU in a population based study, ABI method was used in our study and the sensitivity and specificity of ABI was compared to CDU. Earlier studies have suggested ABI as a reliable method for diagnosis of PVD, with ABI value of 0.9 having $95 \%$ sensitivity. It is also suggested that a single measurement with peripheral Doppler is ideal for identifying majority of the subjects with PVD. In our study, ABI measurement had $88.5 \%$ specificity but the sensitivity was only $70.6 \%$. The low sensitivity indicates that ABI measurement would miss some of the patients with PVD. Conclusion ABI has a very high specificity but the sensitivity is low compared to colour Doppler ultrasound (CDU). Therefore, if $\mathrm{ABI}$ value is abnormal, the diagnosis of PVD is almost certain whereas if a diabetic has a normal ABI value in asymptomatic cases, PVD is unlikely to be clinically significant and probably no further testing is needed. However, in symptomatic cases of PVD with normal ABI, a CDU should be performed before PVD is definitely excluded. An additional problem with $\mathrm{ABI}$ is that if there is vascular calcification, falsely high pressures would be obtained and if fully calcified, pressure recording is not possible. This is reported to occur common in diabetics and in such cases, only CDU should be used to diagnose PVD accurately.

\section{References}

1. King H, Aubert RE, Herman WH. Global burden of diabetes, 1995 - 2025: prevalence, numerical estimates, and projections. Diabetes Care 1998; 21 (9): 1414-31.

2. Prompers L, Schaper N, Apelqvist J et al. Prediction of outcome in individuals with diabetic foot ulcers: focus on the differences between individuals with and without peripheral arterial disease. The Eurodiale Study. Diabetologia 2008; 51 (5): 747-55.

3. Hinchliffe RJ, Andros G, Apelqvist J et al. A systematic review of the effectiveness of revascularisation of the ulcerated foot in patients with diabetes and peripheral arterial disease. Diab Metab Res Rev 2012; 28 (S1): 179-217.

4. Pecoraro RE, Reiber GE, Burgess EM. Pathways to diabetic limb amputation: basis for prevention. Diabetes Care 1990; 13 (5): 513-21.

5. Schaper NC. Lessons from eurodiale. Diab Metab Res Rev 2012; 28 (S1): 21-6.

6. Xu D, Li J, Zou L et al. Sensitivity and specificity of the ankle — brachial index to diagnose peripheral artery disease: a structured review. Vasc Med 2010; 15 (5): 361-9.

7. McDermott MM, Feinglass J, Slavensky R et al. The ankle - brachial index as a predictor of survival in patients with peripheral vascular disease. J Gen Int Med 1994; 9 (8): 445-9.

8. American Diabetes Association. Peripheral arterial disease in people with diabetes. Diabetes Care 2003; 26 (12): 3333- 41.

9. Strandness Jr DE, Bell JW. Peripheral vascular disease: Diagnosis and objective evaluation using a mercury strain gauge. Ann Surg 1965; 161 (Suppl 4): 4.

10. Premalatha G, Shanthirani S, Deepa $R$ et al. Prevalence and risk factors of peripheral vascular disease in a selected South Pakistann population: the Chennai Urban Population Study. Diabetes Care 2000; 23 (9): 1295-300.

11. http://www.japi.org/january2000/22ndPoster Session.htm.

12. Orchard TJ, Strandness DE. Assessment of Peripheral Vascular Disease in Diabetes: Report and Recommendations of an International Workshop Sponsored by the American Heart Association and the American Dia 